\title{
The development of uniportal video-assisted thoracoscopic surgery in São Paulo: from diagnosis to lobectomy
}

\author{
Alexandre de Oliveira, Thais Amarante P. P. Couto \\ Department of Thoracic Surgery, Beneficencia Portuguesa Hospital, Sao Paulo, Brazil \\ Contributions: (I) Conception and design: All authors; (II) Administrative support: A de Oliveira; (III) Provision of study materials or patients: A de \\ Oliveira; (IV) Collection and assembly of data: All authors; (V) Data analysis and interpretation: All authors; (VI) Manuscript writing: All authors; \\ (VII) Final approval of manuscript: All authors. \\ Correspondence to: Alexandre de Oliveira, MD, PhD. Department of Thoracic Surgery, Beneficencia Portuguesa Hospital, Rua Coronel Oscar Porto \\ 208 apto 121B, Sao Paulo, SP, Brazil. Email: alexandretorax@gmail.com; alexandreo@einstein.br.
}

Background: The use of uniportal video-assisted thoracoscopic surgery (VATS) has been increasing worldwide. Our main goal was to describe the evolution of uniportal surgery in the biggest private hospital in Latin America that is located in São Paulo, Brazil.

Methods: This descriptive and retrospective study included patients who underwent uniportal VATS in the thoracic surgical department of Beneficencia Portuguesa Hospital, after being referred to our team to undergo the aforementioned procedure within the period from February 2012 to March 2016. Postoperative management and results were analyzed.

Results: In the thoracic surgical department of Beneficencia Portuguesa Hospital, 454 uniportal VATS surgeries were performed. Of the patients, 287 (65.52\%) were male and 151 (34.48\%) were female, with a mean age of $57.48 \pm 23.4$ years. In December 2015 , we initiated anatomical pulmonary resections (lobectomies and segmentectomies). In the cases of uniportal lobectomies for lung cancer in the initial staging, lymphadenectomy was performed in all the patients, of whom 59 (87\%) had at least seven lymph nodes included in the dissection and confirmed in the pathological anatomy report. Four of the uniportal lobectomy cases were converted to thoracotomy because of bleeding. One patient needed blood transfusion and vasoactive drug administration in the intensive care unit (ICU), and seven patients required pleural procedures (thoracentesis or pigtail catheter) after drainage removal. No operative or perioperative mortality related to the procedure occurred. The main hospital stay was $7.4 \pm 4.3$ days.

Conclusions: The uniportal thoracic procedures performed by our surgical team in São Paulo represent a breakthrough in the surgical treatment of thoracic pathologies in Southeast Brazil and can be offered as a safe and first-choice VATS procedure in our institution.

Keywords: Uniportal; video-assisted thoracic surgery (VATS); lobectomy; minimally invasive thoracic surgery

Submitted Aug 04, 2016. Accepted for publication Sep 30, 2016.

doi: $10.21037 /$ jtd.2016.10.79

View this article at: http://dx.doi.org/10.21037/jtd.2016.10.79

\section{Introduction}

Since video-assisted thoracoscopic surgery (VATS) gained a remarkable place in thoracic surgery, many surgeons started to search, increasingly, for innovations of this technique. Naturally, as years passed, thoracic surgeons realized that the same procedure can be performed with two incisions unlike the classic VATS, which was initially performed with three or four incisions (1-3).

The first cases of uniportal surgery in thoracic sympathectomy reported in 2002 and one of the cases of uniportal VATS in wedge pulmonary resections that were reported by Dr. Rocco from the National Cancer Institute in Naples, Italy, the surgical treatment of pneumothorax, pleural diagnosis, and mediastinal staging motivated us 

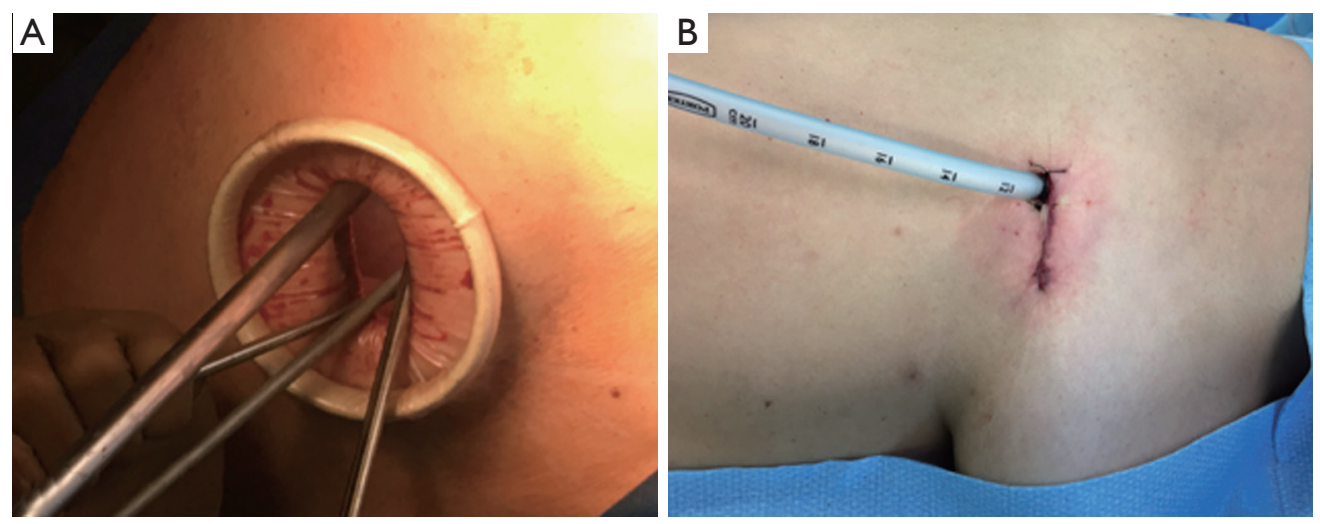

Figure 1 The uniportal procedure: (A) working positioning of instrumentals; (B) chest tube located at the posterior part of the incision, no other incision was made.

to follow on the uniportal VATS surgical journey $(4,5)$. Following this thoughts, we aimed to identify all of the benefits of using the least number of incisions for the thoracic wall, including less postoperative pain, shorter hospitalization time, better esthetic results, high patient satisfaction, and early return to daily activities $(6,7)$.

Rivas's publications on the biggest pulmonary resections such as lobectomies, pneumonectomies, and bronchoplasty, followed by the important results obtained by the Shanghai group from the Pulmonary Hospital of Tongji University, spread throughout the world, encouraging our department to naturally follow the complex procedures performed with the uniportal technique and presenting the same results as those with using VATS with more than one incision (8-11).

That being said, the main goal of this article was to describe the evolution of uniportal surgery that took place in the largest private hospital of Latin America, which is located in São Paulo, Brazil; the difficulties encountered; and the advantages and benefits the method brought to our institution and city.

\section{Methods}

\section{Patients}

A descriptive and retrospective study was conducted with patients who underwent uniportal VATS at the thoracic surgical department of Beneficencia Portuguesa Hospital, São Paulo, Brazil, who were referred to our team with an indication to undergo the aforementioned procedure between February 2012 and March 2016. This highlights the evolution of the uniportal surgery in the city of São
Paulo. All of the patients provided written consent before undergoing the operation, in accordance with the guidelines of the medical ethics committee of our institution.

\section{Surgical technique}

Aside from the sympathectomy cases, all uniportal VATS procedures were performed with the patient under general anesthesia and in the total lateral decubitus position, with single-lung ventilation and similar preparation as that for posterolateral thoracotomy. A 3 to $6 \mathrm{~cm}$ incision was made, and a small $(2.5-6 \mathrm{~cm})$ wound protector (Alexis, Applied Medical, CA, USA) was placed. A 5 or $10 \mathrm{~mm} 30^{\circ}$ scope camera was introduced. Then, an initial thoracoscopy was performed in all of the cases to help with the adaptation of the surgical team to the uniportal technique. No other incision was made, and the cavity was drained through the access incision with a $24-\mathrm{F}$ tubular chest drain (Figure 1).

The patients who were to undergo sympathectomy were intubated with a normal tube and placed in the semi-seated position with their arms opened. Exclusively in these cases, we used a $5.5 \mathrm{~mm}$ trocar next to the axillary region through the single access and performed the procedure (Figure 2).

\section{Statistical analysis}

Categorical variables are reported as $\mathrm{n}(\%)$. Continuous variables are expressed as mean $\pm \mathrm{SD}$ or median depending on the distribution of the variables. Analyses were conducted by using the statistical package SAS University Edition 9.4 for OS X (SAS Institute Inc., Cary, NC, USA). 


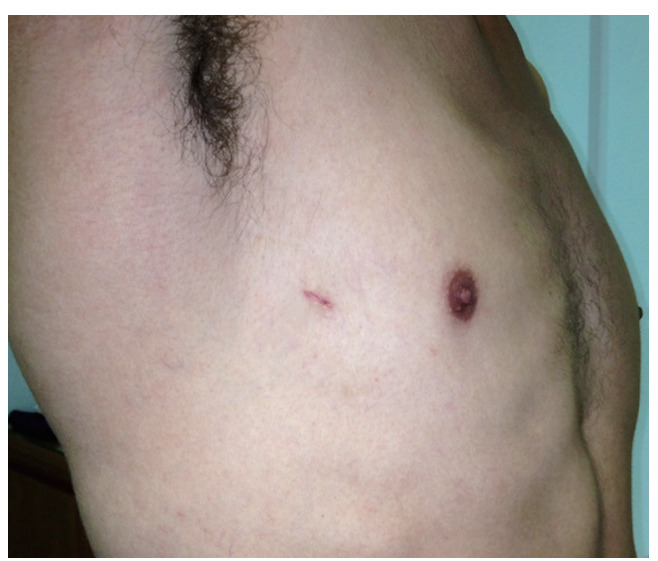

Figure 2 Final result of a uniportal sympathectomy.

Table 1 Uniportal VATS performed in Beneficencia Portuguesa Hospital in São Paulo [2012-2016], divided by major and minor procedures

\begin{tabular}{lc}
\hline Variable & No. of patients [\%] \\
\hline Minor procedures uniportal & $146[32]$ \\
Sympathectomy & $72[16]$ \\
Bullectomy & $65[14]$ \\
Wedge resection & $61[13]$ \\
Pleural biopsy and pleurodesis & $7[2]$ \\
Pleurectomy & $16[4]$ \\
Videothoracoscopy-hemothorax & \\
Major procedures uniportal & $6[1]$ \\
Mediastinal tumor resection & $13[3]$ \\
Segmentectomies & $68[15]$ \\
Lobectomies & 454 \\
\hline
\end{tabular}

VATS, video-assisted thoracoscopic surgery.

\section{Results}

From January 2012 to March 2016, we performed 454 uniportal surgeries at the thoracic surgical department of Beneficencia Portuguesa Hospital, São Paulo, Brazil. The first 47 cases were sympathectomies. Of the patients, 287 $(65.52 \%)$ were male and 151 (34.48\%) were female, with a mean age of $57.48 \pm 23$ years. The procedures performed were divided into minor and major procedure groups
(Table 1).

In December 2014, we initiated the anatomical pulmonary resection cases (lobectomies and segmentectomies) (Figure 3). In the uniportal lobectomies performed for lung cancer in the initial staging, lymphadenectomy was performed in all of the patients. In 59 cases $(87 \%),>7$ lymph nodes were included in the dissection and confirmed in the pathological anatomy report.

Histological data showed that mediastinal mass resection was performed for thymomas in 4 cases (68\%), thymic hyperplasia in 1 case (16\%), and lymphoma in 1 case (16\%).

\section{Perioperative complications and mortality}

Four lobectomy cases were converted to thoracotomy because of bleeding. One patient needed blood transfusion and vasoactive drug administration in the intensive care unit (ICU) but was discharged from the ICU on the sixth postoperative day (Table 2). No operative or perioperative mortality related to the procedure occurred.

\section{Postoperative management}

The chest tube drainage was removed when the secretion volume was $<150 \mathrm{~mL}$. When used, the chest tube was removed after a mean of 3.6 \pm 2.4 days. Among the patients who underwent a lobectomy, four needed thoracentesis after drainage removal because of the high volume of pleural effusion detected on chest radiography. Three patients required insertion of a pleural drainage tube with a pigtail catheter for pneumothorax that occurred after the removal of the tubular chest drain (Table 2). No infections where observed in any of the cases. The mean hospital length of stay was $7.4 \pm 4.3$ days.

\section{Discussion}

Since 2012, the number of surgeries performed with the uniportal technique has increased gradually in spite of its complexity and comprehensiveness. We initiated sympathectomy by using the uniportal technique with $5 \mathrm{~mm}$ $30^{\circ}$ fiber optics.

Confident with the cavity visualization after several procedures to gain access in the sympathetic thoracic chain (sympathectomies), we started to use the uniportal access to diagnose cases and treat pleural collection pathologies such as pleural effusion (recurrent or primary 
A

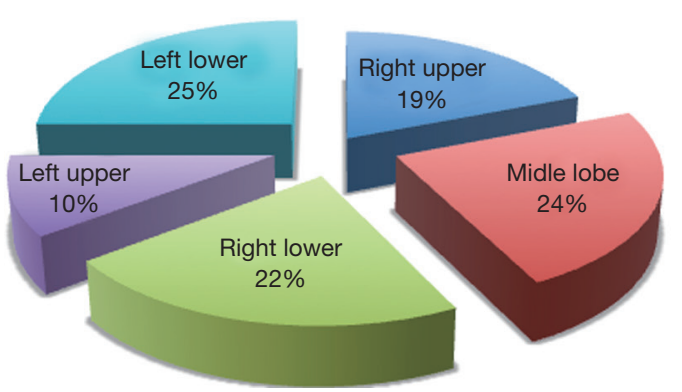

B Uniportal segmentectomies

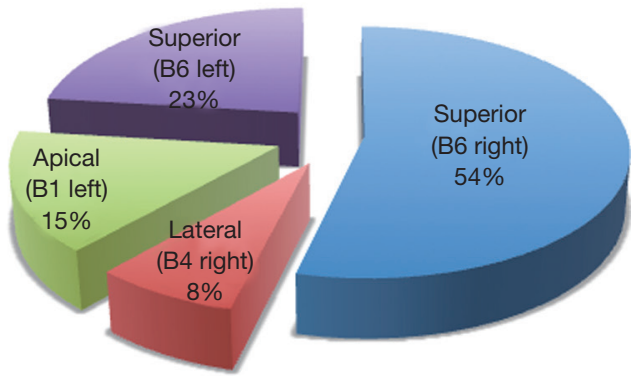

Figure 3 Lung anatomical resections performed by uniportal VATS in our institution: (A) lobectomies by lobes (B) segmentectomies by segments. VATS, video-assisted thoracoscopic surgery.

Table 2 Perioperative and postoperative complications-lobectomies cases

\begin{tabular}{lc}
\hline $\begin{array}{l}\text { Perioperative and postoperative } \\
\text { complications - lobectomies cases (N=68) }\end{array}$ & $\begin{array}{c}\text { No. of } \\
\text { patients (\%) }\end{array}$ \\
\hline Thoracotomy conversion & $4(5.8)$ \\
Pleural effusion (thoracocentesis needed) & $4(5.8)$ \\
Pneumothorax after chest tube removal & $3(4.5)$ \\
Hemodinamic instability by bleeding & $1(1.5)$ \\
Total & $12(17.6)$ \\
\hline
\end{tabular}

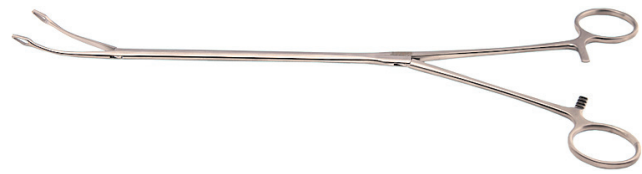

Figure 4 Snake clamp for uniportal procedures.

oncological), pleural metastasis, and definitive spontaneous pneumothorax and hemothorax by closed chest trauma or by postoperative complications in cardiac surgeries.

As our team was involved in the major pulmonary resections with two incisions (one for the camera and the other as utility incision), training centers for uniportal surgeries began emerging, with the Pulmonary Hospital in Shanghai (Tongji University) as the main center.

Apart from being encouraged by the results of the abovementioned smaller procedures, the surgical team lacked the security of seeing an entire department perform major and complex pulmonary resections of the most varied kinds. This need was met after we participated in the first class of the Shanghai Uniportal VATS training program. After the training, we adjusted the surgical staff's positioning in the operational field and the choices of surgical nurses. In addition, we had the opportunity to help in designing an instrument to help in vessel dissection and called it "snake" (Figure 4). With all this improvement, we found a significant decrease in lobectomy time after the eighth case, which decreased from $180 \pm 31.5$ to $120 \pm 30.4$ minutes. Today, after performing 50 lobectomy cases, we can complete a uniportal lobectomy with a naturally regular anatomy in around $80-100$ minutes.

This improvement in our technique to perform uniportal VATS relieved the fatigue of the surgical team, which could lead to the abandonment of a new technique when the learning curve promotes stress on the surgeon.

An important detail was observed in our learning curve regarding patients with obesity. During minor uniportal VATS procedures, these patients developed hypoxemia with one-lung ventilation. In these cases, our department chose not to indicate uniportal major procedures such as lobectomy for patients with a body mass index of $>35 \mathrm{~kg} / \mathrm{m}^{2}$.

In current publications, uniportal VATS lobectomy has proven to be equivalent to multiport thoracoscopic lobectomy in terms of perioperative and postoperative results, with the possible advantages of better pain control and early mobilization of the patient, which we also verified in our patients (12). Similarly, as in our study, the decreases in the amount of time required to attain permanency of pleural drainage and in hospitalization time after uniportal VATS were also shown in some studies (13-15).

As noted by various authors, surgical time was also significantly reduced with the best positioning of the surgical team in the field and better handling of the 
pulmonary hilum, which is easily observed when performing a dissection with the camera view introduced through a uniportal access $(16,17)$.

We believe that training centers and high-standard teaching courses such as the one offered by Charite Hospital of the University of Berlin as the third International Uniportal VATS course, with practical laboratories and non-human live specimens would help promote the professional education of thoracic surgeons who desire to launch their careers and excel in uniportal surgery, specifically in anatomical resections such as lobectomy and segmentectomy.

Today, in our institution, we can safely offer uniportal surgery as the first-choice surgical treatment for patients with early-stage lung cancer and thereby provide patients a shorter recovery time, less postoperative pain, and shorter hospitalization time.

\section{Conclusions}

The uniportal surgery performed by our department in São Paulo represents a breakthrough in the surgical treatment of thoracic pathologies in Southeast Brazil. Its evolution significantly reduced patients' postoperative pain. The technique can be performed safely by members of a welltrained surgical team who are familiarized with the method.

\section{Acknowledgements}

We thank Diego Gonzalez-Rivas and Mahmoud Ismail for introducing us to the field of uniportal VAST.

\section{Footnote}

Conflicts of Interest: The authors have no conflicts of interest to declare.

Ethical Statement: The study was approved by the ethnical committee in Federal University of São Paulo (register number CT2016/84). Written informed consent was obtained from the patient.

\section{References}

1. Roviaro G, Varoli F, Rebuffat C, et al. Major pulmonary resections: pneumonectomies and lobectomies. Ann Thorac Surg 1993;56:779-83.
2. Yim AP, Izzat MB, Liu HP, et al. Thoracoscopic major lung resections: an Asian perspective. Semin Thorac Cardiovasc Surg 1998;10:326-31.

3. McKenna RJ Jr, Houck W, Fuller CB. Video-assisted thoracic surgery lobectomy: experience with 1,100 cases. Ann Thorac Surg 2006;81:421-5; discussion 425-6.

4. Nesher N, Galili R, Sharony R, et al. Videothorascopic sympathectomy (VATS) for palmar hyperhidriosis:summary of a clinical trial and surgical results. Harefuah 2000;138:913-6, 1008.

5. Rocco G, Martin-Ucar A, Passera E. Uniportal VATS wedge pulmonary resections. Ann Thorac Surg 2004;77:726-8.

6. Jutley RS, Khalil MW, Rocco G. Uniportal vs standard three-port VATS technique for spontaneous pneumothorax: comparison of post-operative pain and residual paraesthesia. Eur J Cardiothorac Surg 2005;28:43-6.

7. Rocco G, Brunelli A, Jutley R, et al. Uniportal VATS for mediastinal nodal diagnosis and staging. Interact Cardiovasc Thorac Surg 2006;5:430-2.

8. Gonzalez-Rivas D. VATS lobectomy: surgical evolution from conventional VATS to uniportal approach. ScientificWorldJournal 2012;2012:780842.

9. Gonzalez-Rivas D, Delgado M, Fieira E, et al. Double sleeve uniportal video-assisted thoracoscopic lobectomy for non-small cell lung cancer. Ann Cardiothorac Surg 2014;3:E2.

10. Gonzalez-Rivas D, Fieira E, Delgado M, et al. Is uniportal thoracoscopic surgery a feasible approach for advanced stages of non-small cell lung cancer? J Thorac Dis 2014;6:641-8.

11. Ismail M, Helmig M, Swierzy M, et al. Uniportal VATS: the first German experience. J Thorac Dis 2014;6:S650-5.

12. Shen Y, Wang H, Feng M, et al. Single- versus multipleport thoracoscopic lobectomy for lung cancer: a propensity-matched study†. Eur J Cardiothorac Surg 2016;49 Suppl 1:i48-53.

13. Ng CS. Uniportal VATS in Asia. J Thorac Dis 2013;5 Suppl 3:S221-5.

14. Chen PR, Chen CK, Lin YS, et al. Single-incision thoracoscopic surgery for primary spontaneous pneumothorax. J Cardiothorac Surg 2011;6:58.

15. Akter F, Routledge T, Toufektzian L, et al. In minor and major thoracic procedures is uniport superior to multiport video-assisted thoracoscopic surgery? Interact Cardiovasc Thorac Surg 2015;20:550-5. 
16. Liu CY, Lin CS, Shih CH, et al. Single-port video-assisted thoracoscopic surgery for lung cancer. J Thorac Dis 2014;6:14-21.

Cite this article as: de Oliveira A, Couto TA. The development of uniportal video-assisted thoracoscopic surgery in São Paulo: from diagnosis to lobectomy. J Thorac Dis 2017;9(4):865-870. doi: $10.21037 /$ jtd.2016.10.79
17. Ng CS, Lau KK, Wong RH, et al. Single port videoassisted thoracoscopic lobectomy for early stage non-small cell lung carcinoma. Surgical Practice 2013;17:35-6. 